\title{
Using Flash Software to Make Teaching Courseware for Analog Electronics
}

\author{
Zhang Dongwei \\ Information \& Control Engineering Faculty \\ Shenyang Jianzhu University \\ Shenyang China \\ e-mail: 3730738@qq.com
}

Tan Lisha

Student Work Department

Shenyang Jianzhu University

Shenyang China

tanls@sjzu.edu.cn

\author{
Qi Ning \\ Library \\ Shenyang Jianzhu University \\ Shenyang China \\ e-mail: wind@sjzu.edu.cn
}

\author{
Zhang Shaocheng \\ Informatization Center \\ Liaoning University \\ Shenyang China \\ zhangsc@sjzu.edu.cn
}

\begin{abstract}
Analog electronics course is an important fundamental electric course. It is difficult to study and its content is difficult to comprehend and master. Animated coursewares of PN junction and negative feedback circuits are designed and made using flash technology. They can dynamically demonstrate the motion of carriers, the formation of $P N$ junction and the label of instant polarity. By watching flash courseware students can visually study the abstract and theoretical knowledge. The teaching practice shows that the usage of flash courseware can stimulate the motivation among students and improve the quality and the efficiency of the teaching.
\end{abstract}

Keywords- flash coursewares; analog electronics; PN; junction;

\section{INTRODUCTION}

Analog electronics course is an important fundamental course for the undergraduates of electric profession and non-electric profession to get to know the electronic technologies [1]. This course helps students to learn the basic knowledge, theory and skills in Analog electronic technologies through learning the common electronic components, analog circuits and the way of systemic analysis and design, so that they can find a good basis for further study in analog electronic technologies as well as better application in their specialties. Analog electronics course is so difficult to learn and its content is so hard to comprehend and master that many students are not interested in studying the course and the learning effect is poor [2]. The teachers are confronted with the question of how to in-spire the students' interest and which way should the teachers take to help students master the basic theories and skills of this course [3-6]. Nowadays, many schools use multimedia techonology to make teaching coursewares to assist with teaching in analog electronics course and have received remarkable effect. However, most of the teach-ing courseware in analog electronics course are designed by powerpoint software, which could only show some static words and pictures of circuits instead of the demon-stration of dynamic processes. By contrast, the teaching courseware that designed by flash technology can vividly explain the abstract knowledge and dynamically demonstrate the motion situation of analog circuits, such as the motion of electronic, the effect of the waves of in-put and output and the movement of the currents in the circuit [7]. In this way, it can enhance students' studying of the theoretical knowledge, give students a further understanding of the content and improve the students' skill of learning, so it could receive much more better learning effect.

Flash, produced by American MACROMEDIA Company in 1996, is one of the most widespread software to design web page animation, and it is the powerful 2Danimation-making software to design and edit flash files [8,9]. The predecessor of flash is the Future Splash software produced by the Future Wave Company, which was purchased by Adobe. It is a interact software to design animation. By a series of components consisted by symbols, button, level, frame and screen, it can help people integrate various multimedia materials, such as graphic, sound and animation, make out terse form but rich contents, strong interaction and expressive animations.

\section{MAKING PN JUNCTION}

\section{A. Making a Background Gate}

First, make a background of wood colour and add a white screen on it as the frame of courseware. Just as the Fig.1. Next, it is time to make buttons. The buttons are divided into four situations as bounce, pointer, depress and click. The making steps are described briefly as follows.

(1) Open the "flash CS4", create a new file. After choose a stage, find the button "set" in attribute panel to adjust the size and the colour of the background.

(2) Find and select the order of [insert $] \rightarrow$ [new component], revise its name as "crystal button" and de-fine it as "button" in the dialog box that bounced. Click the 
certain button to enter the inner of element. Then draw the basic graph of "crystal button" in stage.

(3) Set a "ground colour" button in colour panel, adjust the number of colour, use the "gradual change deformation" tool to adjust the location of gradual change in the graph carefully.

(4) Use the rectangle tool to draw a highlight graph. Then use the selected tool to move and combine the highlight graph with the basic colour button, and locate it in the middle. It can be slightly adjust by the direction key on the keyboard.

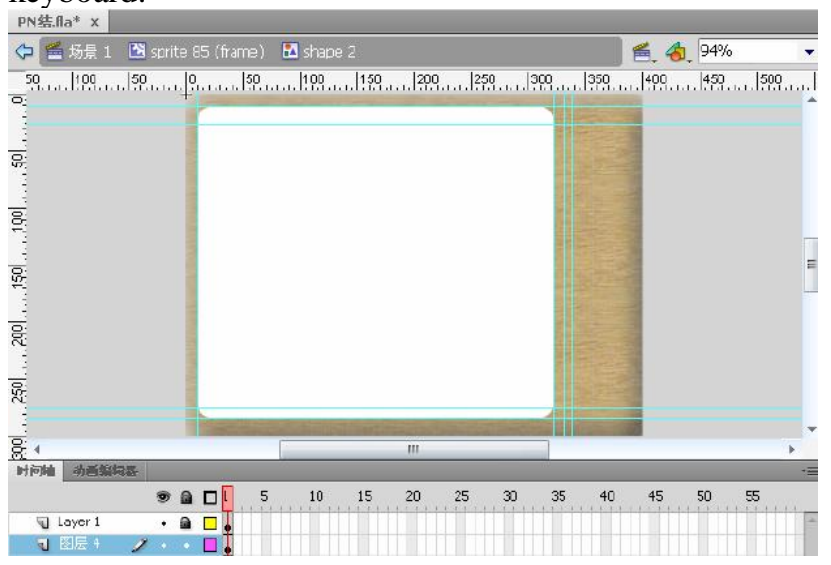

Figure 1. Original background box

Then a crystal button is basically completed. The following work is to make the symbol of school. Create a film edit animation, whose transparency is gradual change. The Alpha change from $100 \%$ to $18 \%$, and then to $100 \%$ as a cycle, so that it can flash continuously in the whole courseware, as is shown in Fig.2.

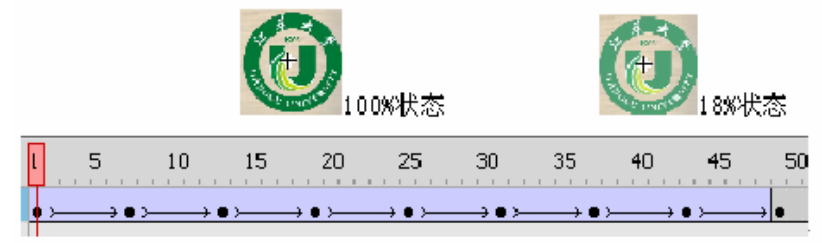

Figure 2. Time axis of symbol

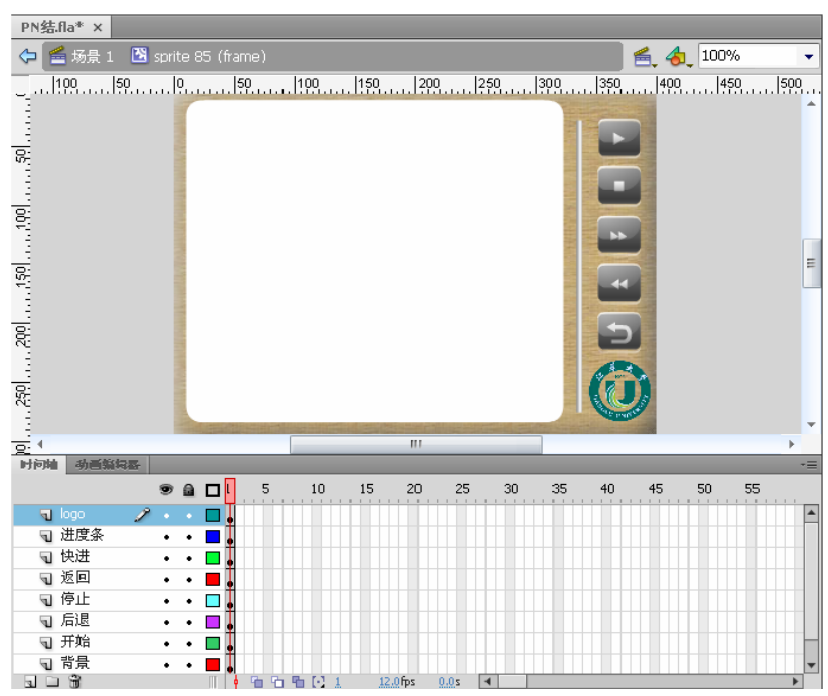

Figure 3. Background box

\section{B. Making the Title}

To produce the process of title "PN Junction" change, animation fade can be made firstly. Alpha changes from $0 \%$ to $100 \%$. Then use arbitrary deformation tools for reducibility, reversal and transparency, create the traditional interpolation, and the process of title change will be formed.

\section{PN Junction}

Figure 4. Process of title change

\section{Fabrication of the Internal Carrier}

Press F8, create a movie clip to fabricate the dynamic process of carrier. Create a new layer; draw the carriers' initial state. Press F6 at frame34, drag all carriers to the needed end state, fill the traditional interpolation, and the movement of carriers will be formed. Then create the layer2, draw the rectangular frame.

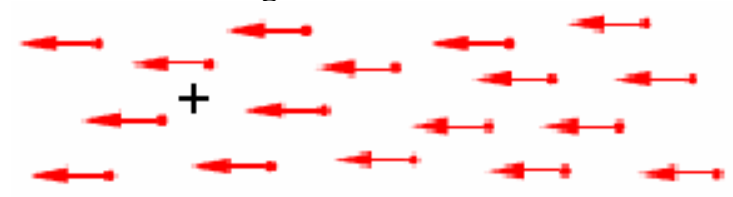

Figure 5. Fabrication of carrier

Let layer 2 be the interface of mask, so layer1 is the masked layer. Then use the frame to cover the carriers, as shown in Fig.6. It is finished after adding a layer to draw the yellow frame.

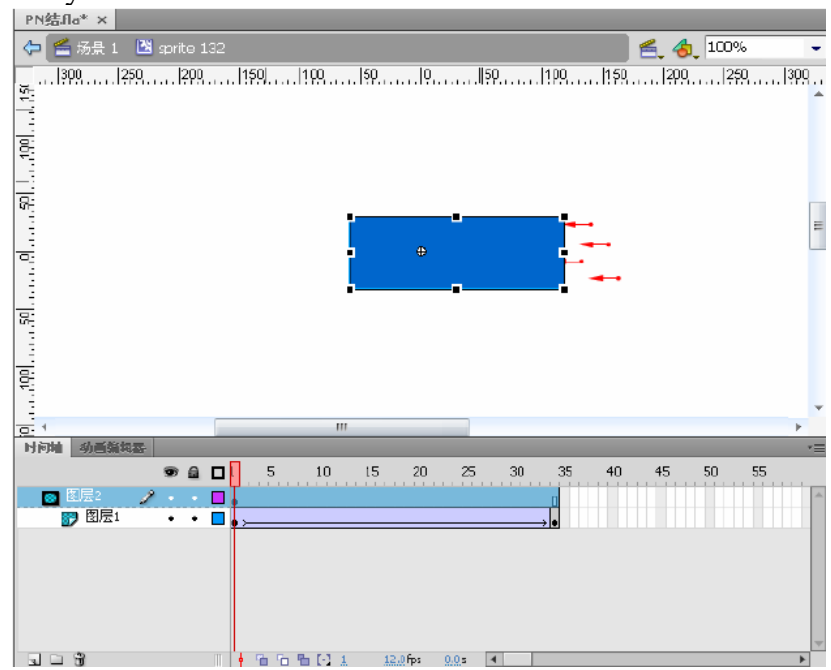

Figure 6. Initial interface of mask

\section{Making the Remotion of the Electronics}

As shown in Fig.7 and Fig.8, the electrons move counterclockwise along the wire. Make components draw the circuit diagram. To achieve the path of electrons moving outside, you can do five electrons moving process, using traditional interpolation can be achieved. Each electron has five steps to go into. Take the second electron for example. 


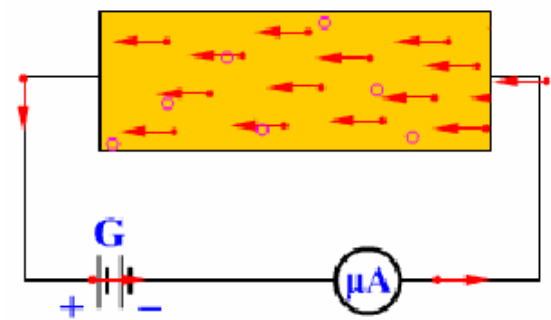

Figure 7. Remotion of the electronics

Step 1: begin from (a) and end at (b) $T$, and form the first traditional interpolation cartoon.

Step 2: begin from (c) and end at (d), and form the second traditional interpolation cartoon.

Step 3: begin from (e) and end at (f), and form the third traditional interpolation cartoon.

Step 4: begin from (g) and end at (h), and form the forth traditional interpolation cartoon.

Step 5: begin from (i) and end at (j), and form the fifth traditional interpolation cartoon. This section is finished.

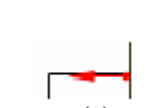

(a)
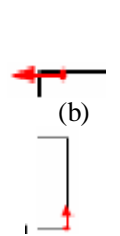

(f)

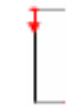

(c)

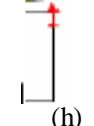

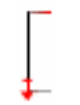

(d)

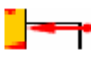

(i)

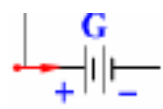

(e)

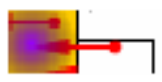

(j)
Figure 8. Graphic combinations

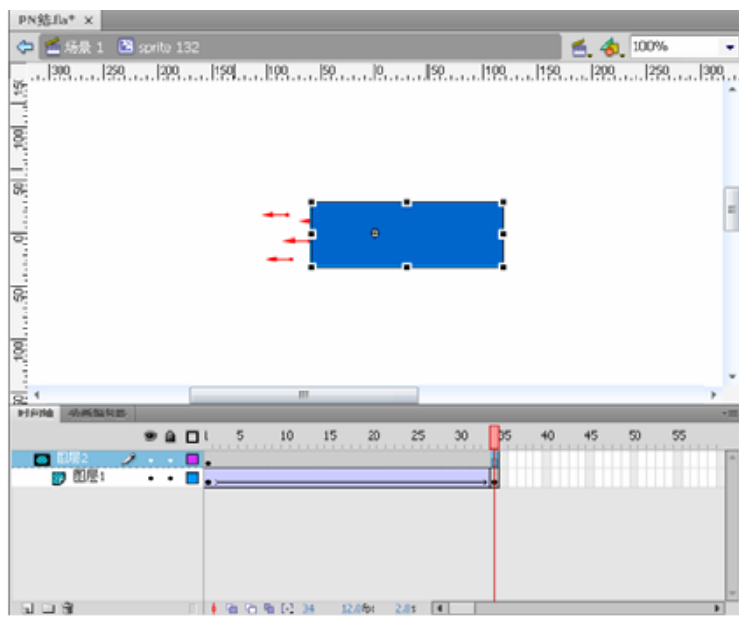

Figure 9. End interface of mask

Use the text tool to write description in each section of the text frame [10], pay attention to the corresponding sound speed. Open the [File] $\rightarrow$ [import] $\rightarrow$ [Import to Library], file the audio into the library. Create a new layer voice", insert the key frame in the end, and then drag it into the "PN junction recording" button. To produce "Thank you for using", from Alpha 0\%, beginning appears below. Press F6 every seven frames, and drag it to the middle. Alpha becomes $100 \%$. Create traditional interpolations, and then maintain 15 frames. Press F5, and finally press F6 separated 7 frames. Drag it to the top of the screen. Alpha changes to $0 \%$, and traditional interpolations are created. This section is finished.

\section{MaKing THE NeGATIVE FeEdB ACK CiRCUITS}

This section uses Flash technology to produce and determine a negative feedback circuit's feedback type and determine the dynamic presentation of negative feedback circuit by using the instantaneous polarity method.

\section{A. Making the Change of Numbers}

This course lists three steps, when each step is carried out, the ball with the corresponding number will be larger, and the others will be smaller, as shown in Fig.9. Firstly, do same things to the three balls. Alpha starts from $0 \%$, and the key frame is inserted after four frames. Then Alpha becomes $100 \%$, and a classic tween is created. Then the ball 1 remains constant to the end of step One, and ball 2 、 ball 3 become smaller after nine frames. Insert the key frame to create the classic tween. Similarly, at the second step, ball 1 becomes smaller, ball 2 becomes larger and ball 3 remains unchanged. At the third step, ball 1 remains unchanged, ball 2 becomes smaller, and ball 3 becomes larger. Do this until the end.
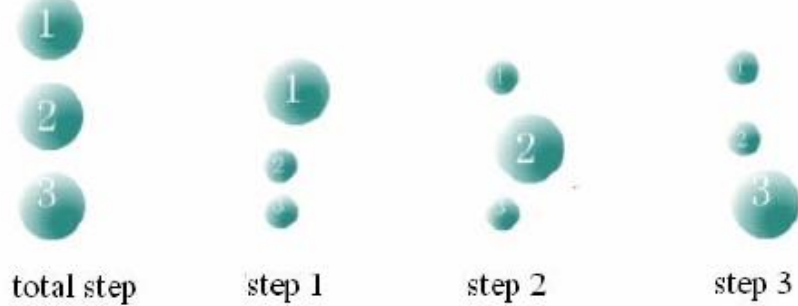

Figure 10. Change of numbers

step 3

Figure 10. Change of numbers

\section{B. Marking the Polarities}

To mark circuit endpoints, balls of the positive and negative charges should be made firstly. The following efforts can be made in controlling the frame of each polarity.
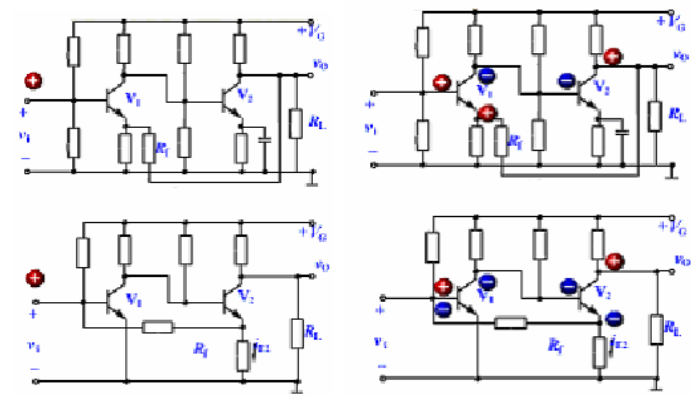

Figure 11. Initial state and end state

Firstly, do the process of moving the NO.1 positive charge. Insert the key frame on the ended position, drag the positive charge to the desired location, and create the traditional interpolation. The scintillation can be made by emptying out a few frames to be blank frames, and then used as the key frame until the end. This is shown in Fig. 12. Similarly, complete the movement, interpolation and maintenance of the positive and negative charges left. 


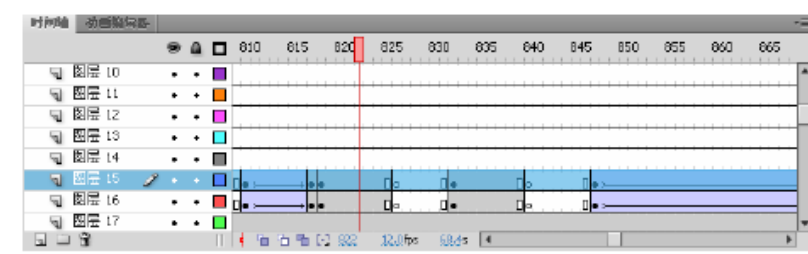

Figure 12. Time axis of charge production

\section{Equations}

The equations are an exception to the prescribed specifications of this template. You will need to determine whether or not your equation should be typed using either the Times New Roman or the Symbol font (please no other font). To create multileveled equations, it may be necessary to treat the equation as a graphic and insert it into the text after your paper is styled.

\section{CONCLUSIONS}

This paper introduces how to setup the distinctive multi-media teaching features also in order to incarnate the characteristics of the multi-media teaching courses, and improve the teaching quantity and efficiency. Furthermore, we make flash-based teaching courseware for the course simulate electronic technique. We make a great deal of simulate electro circuit by the use of flash. As a result, the dynamic shay of electro circuit is realized by the layer structure of electro circuit and relations between parts and the whole are stood ups. The setting of parameters may have its result or functions played with the dynamic state.

\section{REFERENCES}

[1] Huang Chun-Hsiung, Ho PeiChi, and Chung SzuMing, "Computer game programming course for art design students by using flash software, Proc. International Conference on Cyberworlds (CW 2008), IEEE Press, Sep, 2008, pp.710-713, doi: 10.1109/ CW.2008.120.

[2] Mohammed Ahmed, Kadiam Subhash and Chandra Bose S. V. "Simulation, visualization and self-assessment enhanced engineering education: The stiffness matrix method module for structural analysis course", Computers in Education Journal, vol.18, Sep. 2008, pp. 99-106.

[3] Stanley Richard. "Using web based animation software with algorithmic parameters in order to simplify grading while still maintaining oversight of the student's work," Computers in Education Journal, vol. 19, Sept. 2009, p 99-108.

[4] Kuk Kristijan andJovanovic Dragica, "Design and implementation of CoAeLearn modules for personalized game based-learning within Computer Architecture Course," International Journal of Engineering Education, vol.29, Mar. 2013, pp. 620-633.

[5] Stanley Richard, "An efficient way to increase the engineering student's fundamental understanding of particle kinematics and kinetics by utilizing interactive web based animation software," Computers in Education Journal, vol. 18, Sep. 2012, pp. 23-41.

[6] Gao Zhenguo, Liu Sheng and Liang Lihua, "Virtual hydraulic experiments in courseware: 2D virtual circuits and 3D virtual equipments," Computer Applications in Engineering Education, $v$ 19, Jun. 2011, pp. 315-326.

[7] Ngalamou Lucien and Myers Leary, "A macromedia flash-based teaching aid for digital electronic tutoring," International Journal of Electrical Engineering Education, vol.47, Apr. 2010, pp. 104-119.

[8] Ngalamou Lucien and Myers Leary,"A Macromedia Flash-based teaching aid for digital electronic tutoring," International Journal of Electrical Engineering Education, vol.46, Feb. 2015, pp. 104-119.

[9] Ubul Kurban, "Flash based platform for teaching stored programcontrolled switching courses," International Journal of Emerging Technologies in Learning, vol.9, Apr. 2014, pp. 14-19.

[10] Li Lin, Juarez Jaime Israel and Yang Yonggao, "Work in progress: Programming concept visualization using flash animations," Proc. ASEE Annual Conference and Exposition, IEEE Press, Dec. 2012, pp.19-29. 